\title{
Comparison of scrape-off layer profiles in outboard- versus inboard-limited plasmas in Tore Supra
}

\author{
M Kočan* and J P Gunn \\ CEA, IRFM, F-13108 Saint-Paul-lez-Durance, France \\ * Present address: Max-Planck-Institut für Plasmaphysik, Bolzmannstr. 2, D-85748 \\ Garching, Germany \\ E-mail: martin.kocan@ipp.mpg.de
}

\begin{abstract}
In the Tore Supra tokamak, the scrape-off layer profiles of discharges limited either on the inboard or on the outboard side are compared. Inboard-limited discharges are characterized by substantially longer SOL e-folding lengths of ion and electron temperatures and electron density measured near the top of the plasma, providing strong evidence that the ion and electron energy transport across the SOL is enhanced on the outboard side, similar to the particle transport. The parallel heat flux density extrapolated to the last closed flux surface is found to be inversely proportional to its e-folding length in the SOL. The outboard limiters thus receive higher and more peaked heat loads than the inboard limiters. These results are important for the optimization of the plasma start-up scenario and design of the first wall in ITER.
\end{abstract}

PACS 52.25.Fi, 52.25.Xz, 52.35.Ra, 52.55.Fa, 52.70.Ds

\section{Introduction}

In tokamaks, the intermittent expulsion of magnetic-field-aligned plasma filaments from the last closed flux surface (LCFS) is responsible for a large fraction of the effective radial transport to the wall [1-6]. Filament propagation appears to be governed by a ballooning-type instability in the unfavourable magnetic curvature region on the low field side. As the filaments propagate outward, they also expand freely along the magnetic field lines, driving pressure-driven field-direction-independent parallel flows. Mach probe measurements in many divertor tokamaks (e.g. [7] and references therein) show flow patterns consistent with this model (i.e. stagnant flow poloidally approximately halfway between the outer midplane and the X-point), in which the filaments can be seen as an important particle source in the scrape-off layer (SOL). Similar observations in limiter tokamaks [8-12] prove that enhanced radial particle transport on the outboard side of the plasma is a universal phenomenon, independent of the presence of an X-point divertor. The enhancement of the radial particle transport on the outboard side of the limiter plasmas was confirmed by the 3D fluid turbulence code TOKAM-3D [13]. When the plasma contact point is on the inboard limiters, the filaments propagate freely out to the wall, filling all the available volume outside the LCFS and leading to a broad SOL with nearly flat density profiles. On the other hand, it was demonstrated [12] that outboard modular limiters suppress the radial particle transport when they are near the LCFS, leading to a very thin SOL. It is probable [8] that the limiters act as simple mechanical barriers that intercept the parallel particle flux in the region in which the enhanced radial convection. 
The poloidal asymmetry in the radial particle and electron energy transport has been occasionally studied in limiter as well as divertor tokamaks using Langmuir probes located at different poloidal angles (e.g. [8, 10-11, 14-16]) and/or by changing the magnetic field topology (e.g. [16-18]). Measurements in ASDEX and PDX in a double-null divertor configuration have demonstrated that the outer scrape-off layer carries about five times more particles and energy to the divertor as the inner scrape-off layer, providing strong evidence that the cross-field particle and energy transport is larger on the outboard side of the plasma [19]. Similar observations were reported from T-10 and were associated with balloning-like cross-field transport asymmetries [9]. LaBombard and Lipschultz [8] reported a strong poloidal variation of the SOL electron density $n_{e}$ measured in limiter discharges in Alcator C. The maximum $n_{e}$ was measured at the top and the bottom of the plasma and the minimum at the inboard side with the largest density e-folding lengths near the outboard midplane. The poloidal variation of the electron temperature $T_{e}$ was not as dramatic as for the electron density and $T_{e}$ seemed to be almost independent of radius within the data scatter. LaBombard and Lipschultz clearly identified the necessity of a poloidally varying radial convection process (which is now known to be driven by the interchange instabiltiy [1-5]) to account for the measured density profiles. In the limiter tokamak DITE [10], the SOL $T_{e}$ was also found to be a weak function of the poloidal angle but, in contrast to [8], the maximum of the SOL $n_{e}$ was observed near the outer midplane. Stagnant flow measured at this location was associated with the enhanced particle diffusion at the outboard side of the plasma. Similar poloidal asymmetry of the particle transport across the SOL was observed in the FTU tokamak [11]. Later on, measurements in Tore Supra [16] and JET [17] limited discharges demonstrated that the SOL $T_{e}$ profile broadens significantly when the plasma contact point is shifted from the outboard to the inboard side, demonstrating that the electron energy transport across the SOL is also enhanced on the outboard side, similar to the particle transport. LaBombard et al. [18] reported a factor of 4 larger electron pressure e-folding length together with at least an order of magnitude higher fluctuation level of the ion saturation current measured in the outboard SOL compared to the inboard SOL in the Alcator C-Mod double-null discharges, providing again a clear evidence for the existence of a strong poloidal asymmetry in the radial particle and energy transport. These observations were recently reproduced in [20].

Although it is quite intuitive that the radial ion energy transport should be also enhanced on the plasma outboard side (as the magnetic-field-aligned plasma filaments carry into the SOL higher temperatures than the plasma background), experimental evidence of the poloidal asymmetry of the ion energy transport across the SOL is not available.

In this paper, new measurements of the e-folding lengths of the SOL ion and electron temperatures, electron density, and the parallel heat flux density carried out in the limiter tokamak Tore Supra [21] using a retarding field analyzer (RFA) are reported. Improving upon earlier similar studies, we analyze a larger dataset with a broad range of the main plasma parameters and measure the SOL ion temperature $T_{i}$ - an essential, yet rarely measured [2248], parameter for the interpretation of Langmuir probe measurements and SOL modelling. We demonstrate for the first time, that the poloidal asymmetry in the radial ion energy transport is similar to that of the particle and electron energy transport.

The measurements reported here, apart from their contribution in understanding the particle and energy transport in the SOL, are also important for optimizing the design of the first wall and the plasma start-up scenario in ITER. One of the options for the plasma start-up in the existing ITER design consists of two outboard limiters (the possibility to eliminate the limiter modules in favour of plasma start-up on the first wall was recently investigated in [49]). The limiters would define the plasma last closed flux surface for about 30 seconds before the X-point divertor configuration is established [50]. The start-up plasma would in this case therefore be characterized by a configuration similar to that of large limiter 
tokamaks. Our measurements indicate that due to the longer particle and energy e-folding lengths in the inboard-limited discharges, power handling in ITER start-up phase would be significantly easier if the inboard wall were used for start-up rather than outboard limiters.

\section{Experimental set-up}

Ohmic discharges with the plasma contact point either on the inboard limiters (referred to as "inboard-limited discharges") or on the outboard limiters ("outboard-limited discharges") are studied. The inboard limiters (IL) consists of six bumper limiters of toroidal width $20^{\circ}$ (corresponding to $0.55 \mathrm{~m}$ at the inner midplane), separated by $60^{\circ}$. The outboard limiters (OL) consist of six radially movable limiters: antenna protection limiter at toroidal angle of $140^{\circ}$ (toroidal width of $34 \mathrm{~cm}$ ), three ICRH antennae limiters at $40^{\circ}, 100^{\circ}$, and $280^{\circ}$ (toroidal width of $63 \mathrm{~cm}$ ), and two lower hybrid antennae limiters at $320^{\circ}$ and $340^{\circ}$ (toroidal width of $101 \mathrm{~cm}$ ). Further details of the Tore Supra limiters can be found e.g. in [51, 52]. In this experiment the outboard limiters were aligned (i.e. located at the same major radius). The degree to which the discrete inboard and outboard limiters approximate a toroidally continuous limiter will be discussed in detail in section 3.2. Both $I_{p}$ and $B_{t}$ are oriented clockwise looking from the top of the torus. The working gas is deuterium. The database comprises 22 inboard- (including 4 detached) and 5 outboard-limited measurements (including 1 detached), figure 1 and table 1 .
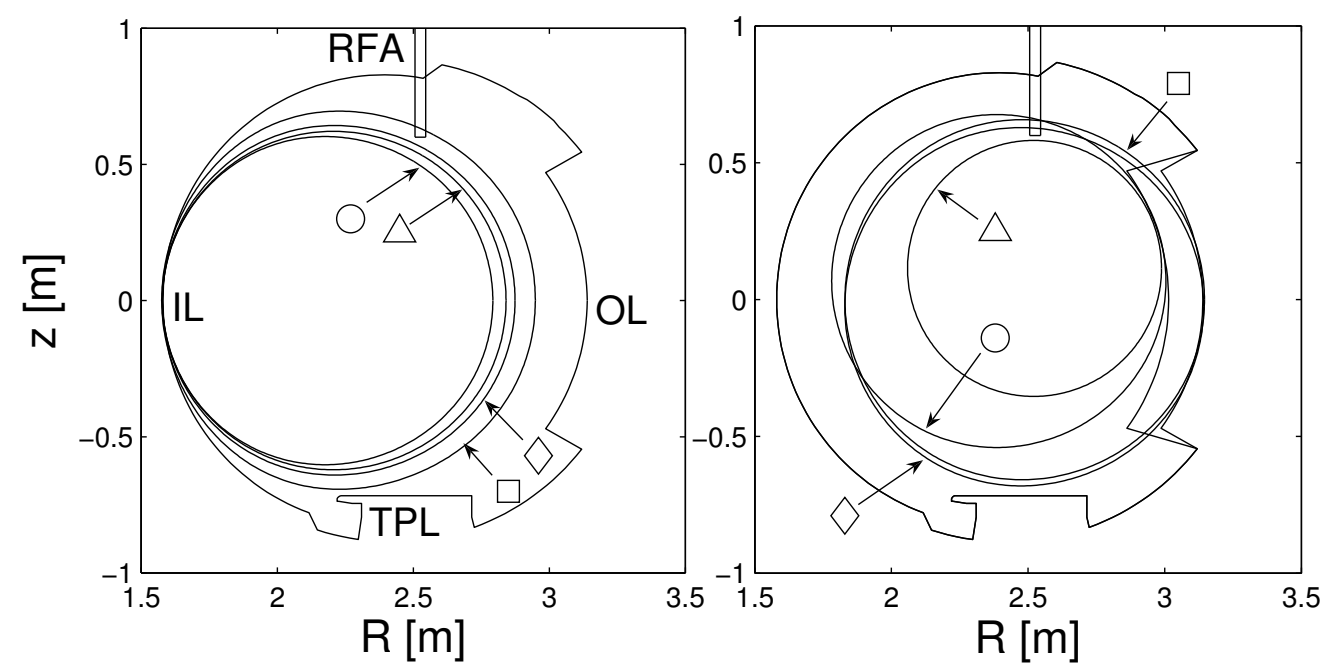

Figure 1. Poloidal plasma cross sections included in the database. Left: inboard contact point. Right: outboard contact point. IL: inboard bumper limiters, OL: radially movable outboard limiters, TPL: toroidal pump limiter. $R F A$ is located at $R=2.53 \mathrm{~m}$ and moves vertically. The symbols associated with the cross-sections are used in figures 4 to 7. 
Table 1. Macroscopic parameters of the database discharges. From top to bottom: minor radius, major radius, volume-averaged density, plasma current, safety factor at the minor radius, toroidal magnetic field at magnetic axis, radiated power fraction and ohmic input power.

\begin{tabular}{lll}
\hline parameter & inboard & outboard \\
\hline$a[\mathrm{~m}]$ & $0.60-0.68$ & $0.47-0.65$ \\
$R[\mathrm{~m}]$ & $2.18-2.26$ & $2.39-2.52$ \\
$\left\langle n_{e}\right\rangle\left[10^{19} \mathrm{~m}^{-3}\right]$ & $0.9-4.7$ & $1.4-5$ \\
$I_{p}[\mathrm{MA}]$ & $0.4-1.2$ & $0.3-0.7$ \\
$q_{a}$ & $3.4-8.5$ & $3.5-9.2$ \\
$B_{t}[\mathrm{~T}]$ & $3.2-4.1$ & $3.5-3.7$ \\
$f_{\text {rad }}$ & $0.3-\sim 1$ & $0.4-0.8$ \\
$P_{\text {ohm }}[\mathrm{MW}]$ & $0.3-1.2$ & $0.3-0.7$ \\
\hline
\end{tabular}

"Detachment" refers here to a steady state with poloidally symmetric radiation shifted inside the LCFS to about $60 \%$ of the minor radius $a$. A steady-state detached phase was reached by increasing the plasma density at constant $I_{p}$. The gas injection rate was controlled in real time by feedback on the radiated power fraction $f_{\text {rad }}$ to reach $f_{\text {rad }} \approx 1$. Plasma detachment was identified visually by two CCD cameras viewing the plasma through a tangential port, and quantitatively by bolometer arrays.

SOL $T_{i}, T_{e}$, and the parallel ion saturation current density $j_{\text {sat }}$ were measured simultaneously from both directions along the magnetic field lines by a bidirectional RFA [46]. The RFA is located at $R=2.53 \mathrm{~m}$ and moves vertically, figure 1 . In this experiment, several novel improvements of the dynamic range of the RFA measurements were employed and described in detail in [18].

$T_{i}$ is calculated as $\left(T_{i}^{H F S}+T_{i}^{L F S}\right) / 2$ [53], HFS/LFS indicating respectively the analyzer intercepting magnetic field lines coming from the high field side or the low field side (typically, each side of the RFA measures different $T_{i}$, which, as shown in [53], is associated with the perturbing effect of the probe combined with the parallel plasma flows). A model for the HFS/LFS asymmetry of $T_{e}$ is not yet available so we arbitrarily choose to estimate $T_{e}$ as $\left(T_{e}^{H F S}+T_{e}^{L F S}\right) / 2$. Electron density and the parallel heat flux density are calculated as $n_{e}=j_{\text {sat }} /\left[0.35 e^{1.5} \sqrt{\left(T_{i}+T_{e}\right) / m_{i}}\right] \quad[54]$ with $j_{\text {sat }}=\sqrt{j_{\text {sat }}^{H F S} \cdot j_{\text {sat }}^{L F S}} \quad$ [55], and $q_{/ /}=\gamma T_{e} j_{\text {sat }}$, respectively. The total (ion and electron) heat transmission coefficient $\gamma$ is calculated as [56]:

$$
\gamma=\frac{2.5 T_{i}}{T_{e}}+2-0.5 \ln \left[\left(2 \pi \frac{m_{e}}{m_{i}}\right)\left(1+\frac{T_{i}}{T_{e}}\right)\right] .
$$

Secondary electron emission was neglected in Eq.(1) so that the equation gives the lower limit of $\gamma[56,57]$. 


\section{Experimental results}

The results are separated into two sections. In section 3.1 the poloidal asymmetry of the radial particle and energy transport is studied in terms of the e-folding lengths. Section 3.2 focuses on the relationship between the parallel heat flux density and its e-folding length and shows that poloidal asymmetry of the radial transport may account for significant differences in the power footprints on the inboard and the outboard limiters.

\subsection{The e-folding lengths}

Figure 2 illustrates the radial profiles of $T_{i}, T_{e}, n_{e}$, and $q_{/ /}$measured in the selected inboard- and outboard-limited discharges characterized by similar macroscopic parameters (table 2). The former are characterized by a factor of $\sim 3-4$ longer e-folding lengths than the latter. From a simple balance between the parallel and perpendicular particle and energy flows into a flux tube in the SOL (see e.g. [58]) it follows that longer e-folding lengths in the inboard-limited discharge could be either due to longer magnetic connection length $L_{c o n}$ or due to the enhanced particle and energy transport across the outboard LCFS (since both measurements are performed at very similar poloidal angles with respect to the plasma, the effect of the flux expansion on the e-folding lengths can be neglected). Figure 3 shows for both discharges $L_{c o n}$ given by the total length of the magnetic field line intersecting the RFA trajectory across the SOL. The variations in $L_{c o n}$ across the SOL due to specific limiter geometry and safety factor are only a small fraction of the total connection length and thus have negligible influence on the e-folding lengths $\left(\lambda \sim \sqrt{L_{c o n}}\right.$ [59]). As seen from figure 3, the inboard-limited discharge is, in fact, characterized by $20-30 \%$ shorter $L_{c o n}$ compared to the outboard-limited discharge. The difference in the e-folding lengths can be, therefore, associated with enhanced radial particle and energy transport on the outboard side of the plasma.

The variation of the density e-folding lengths with the plasma contact point in figure 2 is fully consistent with the results from Alcator $\mathrm{C}$ [8] obtained in a different experimental arrangement. In Alcator $\mathrm{C}$, the density profiles were measured between full poloidal limiters, directly observing the radial propagation of plasma on the outboard side. This is equivalent of what would be measured between the outer limiters in Tore Supra if there were probes on the outer midplane. In our experiment the modular outboard limiters are blocking the parallel transport of the radially-propagating field aligned filaments for the outboard-limited discharges, leading to a thin SOL on top of the torus (this happens if the poloidal extent of the modular poloidal limiters is larger than the extent of the region in which the enhanced radial convection occurs).

Note that in Tore Supra the clearance between the LCFS and the limiters opposite the contact limiter is almost an order of magnitude larger compared to JET limited discharges [18] so that the e-folding lengths measured in the Tore Supra SOL correspond to natural decay lengths associated with free radial transport of the plasma.

An important point to address is whether the plasma is not perturbed by the insertion of the probe and if the e-folding lengths measured by the RFA are similar to those prevailing in its absence. A simple model proposed by Stangeby [60] allows for rough estimate of the degree of the probe disturbance by comparing the ambipolar collection length of the probe wake, $L_{c o l}$, with $L_{c o n}$. The probe disturbance is expected to be significant if $L_{c o l}>L_{c o n}$, in which case the probe establishes a new, shorter, connection length. $L_{c o l} \cong d^{2} c_{s} / 8 D_{\perp}$ where $d=0.04 \mathrm{~m}$ is the RFA diameter, $c_{s}=\sqrt{e\left(T_{i}+T_{e}\right) / m_{i}}$ is the isothermal ion sound speed (with temperatures in electron volts) and $D_{\perp}$ is the cross-field particle diffusivity. Assuming $D_{\perp}=1$ 
$\mathrm{m}^{2} \mathrm{~s}^{-1}, L_{c o l}<11 \mathrm{~m}$ for the inboard-limited discharge and $<14 \mathrm{~m}$ for the outboard-limited discharge across the SOL. This is shorter than $L_{c o n}$ between either side of the RFA and the strike zone for both the inboard- as well as outboard-limited discharge, meaning that the perturbing effect of the probe does not need to be considered.
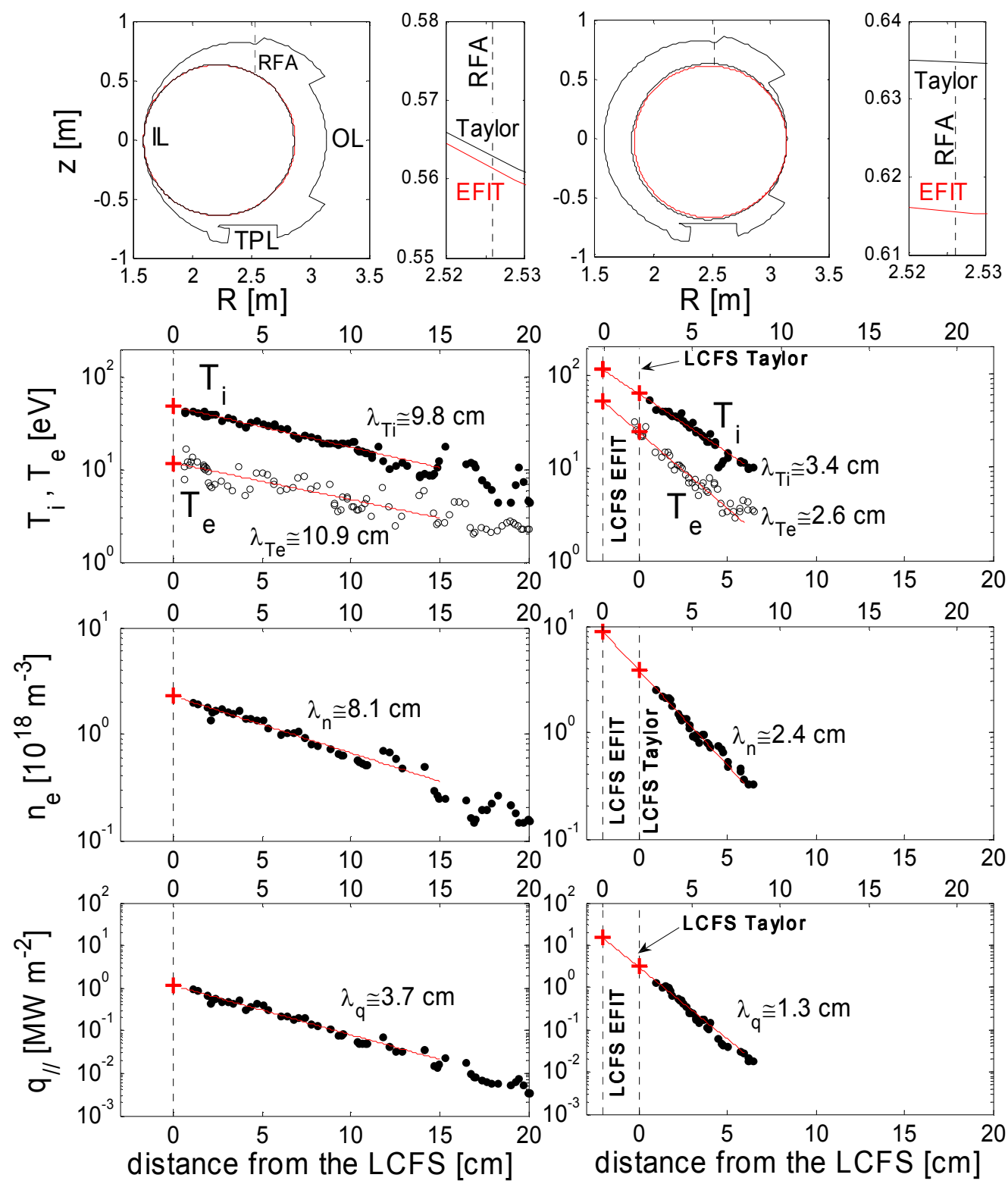

Figure 2. SOL profiles measured by RFA. Left: inboard-limited discharge (\#42390). Right: outboard-limited discharge (\#42460). From top to bottom: LCFS with the magnified region of the RFA location, ion and electron temperatures, electron density, parallel heat flux density with e-folding lengths. SOL profiles are plotted against the distance from the LCFS at the probe location. Two LCFS reconstructions (Taylor, EFIT) are shown. Macroscopic parameters of the discharges are stated in Table 2. 
Table 2. Macroscopic parameters of the discharges from figure 2. The plot symbols correspond to poloidal cross sections from figure 2. The color code is used in figures 4-7. Inboard / outboard indicate the plasma contact point.

\begin{tabular}{lll}
\hline parameter & inboard & outboard \\
\hline discharge number & 42390 & 42460 \\
symbol & $\square$ & $\diamond$ \\
$a[\mathrm{~m}]$ & 0.65 & 0.65 \\
$R[\mathrm{~m}]$ & 2.23 & 2.49 \\
$\left\langle n_{e}\right\rangle\left[10^{19} \mathrm{~m}^{-3}\right]$ & 2.1 & 2.0 \\
$I_{p}[\mathrm{MA}]$ & 0.6 & 0.7 \\
$q_{a}$ & 5.4 & 5.4 \\
$B_{t}[\mathrm{~T}]$ & 3.2 & 3.7 \\
$f_{\text {rad }}$ & 0.4 & 0.6 \\
$P_{\text {ohm }}[\mathrm{MW}]$ & 0.5 & 0.5 \\
\hline
\end{tabular}

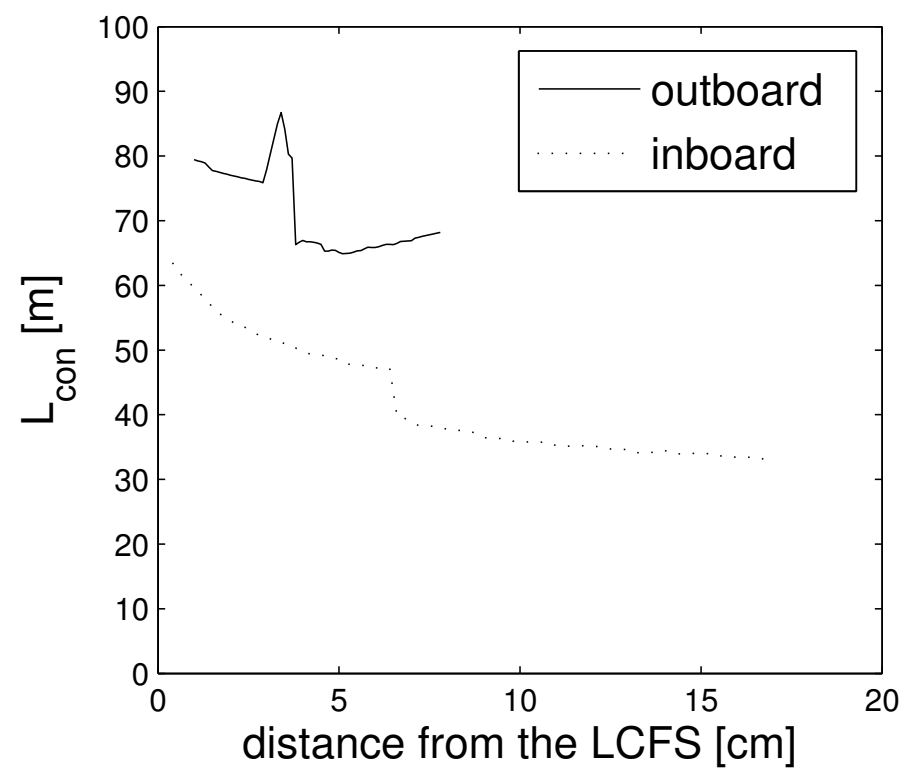

Figure 3. Radial profiles of the magnetic connection length between the strike points at the limiters for the discharges from figure 2. $L_{c o n}$ is calculated for the magnetic field lines intercepting the RFA trajectory across the SOL. Inboard / outboard indicate the plasma contact point.

Note that for the outboard-limited discharges the LCFS at the RFA location calculated by the EFIT poloidal field reconstruction (referred to as "EFIT LCFS") was found to be shifted inward by up to $\sim 2 \mathrm{~cm}$ compared to the LCFS given by the Taylor extrapolation of the 
magnetic flux measurements [61] (referred to as a "Taylor LCFS"). This leads to a large uncertainty in the LCFS values. It is not yet clear which magnetic reconstruction is more reliable. However, for deep reciprocations performed by other Langmuir probes in the outboard discharges a sharp increase in the gradients of $T_{e}$ and $j_{\text {sat }}$ (possibly related to the transition between the SOL and the confined region) coincides with the Taylor LCFS. Therefore, in what follows Taylor LCFS is considered. For most inboard-limited discharges EFIT and Taylor LCFS overlap within a few millimeters. It is important to notice that the uncertainty of the LCFS position is almost inconsequential for the calculation of the e-folding lengths. On the other hand, a factor of 3-4 higher $q_{/ / L C F S}$ is obtained in the outboard-limited discharges for the EFIT LCFS compared to the Taylor LCFS.

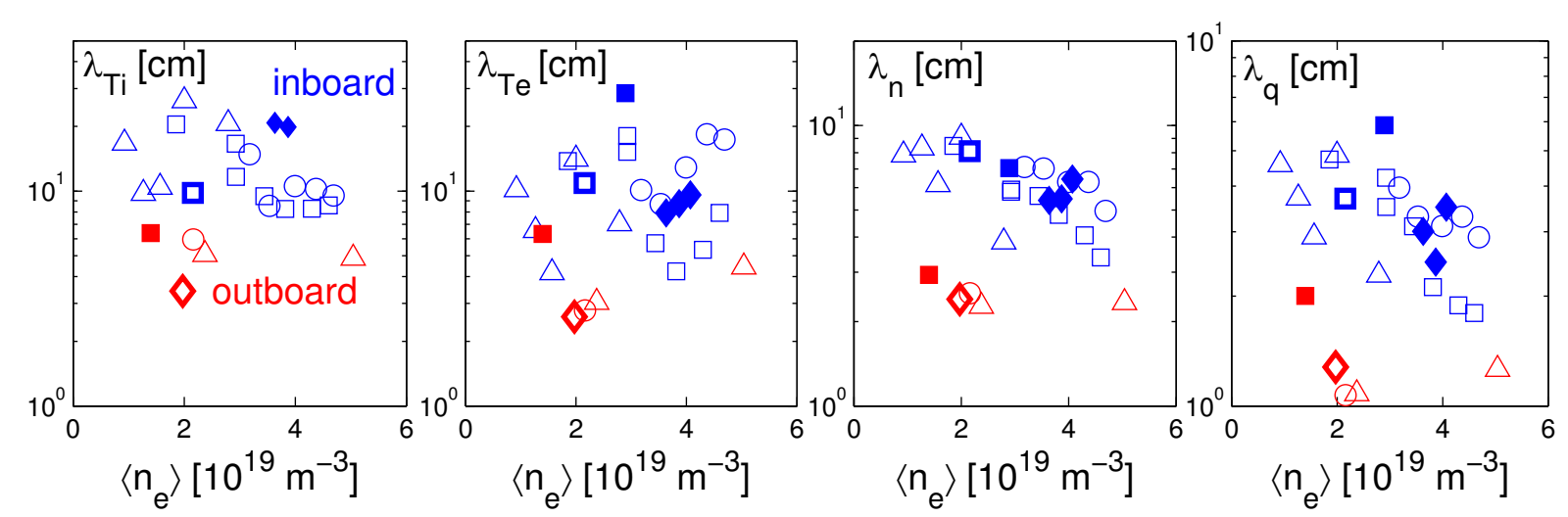

Figure 4. The e-folding lengths of the SOL ion and electron temperatures, electron density and the parallel heat flux density (from left to right) plotted against the volume-averaged plasma density. Inboard / outboard indicate the contact point. Symbols correspond to poloidal cross sections from figure 1. Full symbols: detached discharges. $\lambda_{T i}$ measured in two detached inboard discharges is almost infinite and does not appear on the graph. Discharges from figure 2 are indicated by thick symbols.

Figure 4 shows the e-folding lengths of the ion and electron temperatures $\left(\lambda_{T i}, \lambda_{T e}\right)$, electron density $\left(\lambda_{n}\right)$, and the parallel heat flux density $\left(\lambda_{q}\right)$ for all database discharges, plotted against the volume-averaged plasma density $\left\langle n_{e}\right\rangle$. The e-folding lengths are calculated from the portion of the SOL profile measured between the LCFS and the next closest limiter. As figure 2 has already hinted, the outboard-limited discharges are generally characterized by shorter e-folding lengths compared to the inboard-limited discharges. For a given poloidal configuration several macroscopic parameters are varied simultaneously, figure 5, and their influence on the e-folding lengths thus cannot be decoupled.
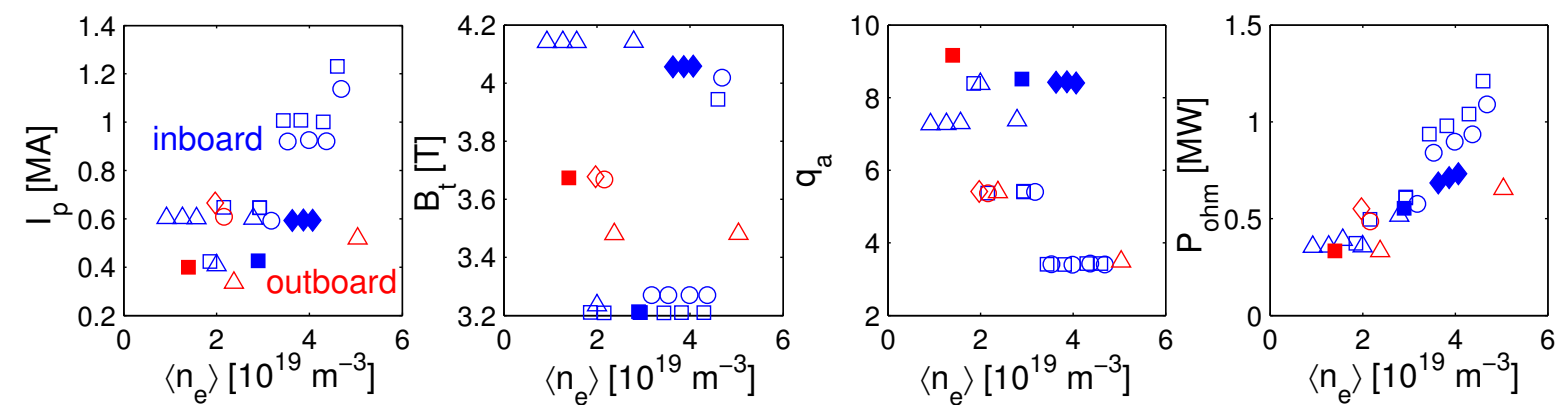
Figure 5. From left to right: plasma current, toroidal magnetic field at magnetic axis, safety factor at the minor radius and ohmic input power plotted against the volume-averaged plasma density. Inboard / outboard indicate the contact point. Symbols correspond to poloidal cross sections from figure 1. Full symbols: detached discharges.

The rarity of SOL $T_{i}$ measurements in tokamaks makes interesting the comparison of $\lambda_{T i}$ and $\lambda_{T e}$. For the database discharges $\left\langle\lambda_{T i} / \lambda_{T e}\right\rangle=1.4 \pm 0.5$ (this mean value does not include two detached inboard-limited discharges with almost infinite $\lambda_{T i}$ ). Similar values were measured e.g. by Uehara et al. in the JFT-2M tokamak [35], in contrast to a simple model for the radial dependence of SOL temperatures [62] which predicts $\lambda_{T i} \rightarrow \infty$ and $\lambda_{T i} / \lambda_{T e} \rightarrow \infty$. The model assumes that the parallel ion energy losses at the limiter are balanced by the convected ion energy flux into the SOL (so that the ions are not cooled passing through the SOL) while the parallel electron energy losses at the limiter are higher than the electron energy flux across the LCFS because of the cooling effect of the Debye sheath on electrons so that $T_{e}$ decreases with radius in the SOL. The model neglects volumetric ion losses in the SOL such as ion collisions with electrons and neutrals which might be the reason why, in contrast to the measurements, it predicts radially constant $T_{i}$ in the SOL. For example, for the database discharges, the ratio of the parallel ion transit time through the SOL to the ion-electron thermalization time $R_{t h}=\tau_{/ /}^{i} / \tau_{t h}^{i e} \quad$ (with $\tau_{/ /}^{i} \propto L_{c o n} / \sqrt{T_{i}+T_{e}}$ and $\left.\tau_{t h}^{i e} \propto T_{e}^{3 / 2} / n_{e}\right)$ evaluated just inside the LCFS varies from 0.3 to 40 (and is $>1$ for most discharges), meaning that significant energy coupling is provided between ions and electrons and can strengthen the similarity between $T_{i}$ and $T_{e}$ profiles in the SOL.

For the database discharges $T_{i}=5-65 \mathrm{eV}, T_{e}=5-30 \mathrm{eV}$ and $T_{i} / T_{e} \cong 1-5$ at the LCFS. The lowest $T_{i}, T_{e}$ and $T_{i} / T_{e}$ are measured at highest plasma densities and in detached plasmas. The decrease of $T_{i}$ and $T_{e}$ with the plasma density is consistent with similar measurements reported from a number of tokamaks [31-33, 37, 39, 42, 45, 48].

\subsection{Parallel heat flux density and its relation to $\lambda_{q}$}

As demonstrated in figures 2 and 4, the poloidal asymmetry in the particle and energy transport makes $\lambda_{q}$ strongly dependent on the location of the plasma contact point. In addition, as seen from figure 6 , at the LCFS $q_{/ / L C F S} \propto \lambda_{q}^{-1}$. The power flux profiles with the highest amplitude and peaking are thus measured in outboard-limited discharges (an exception to this rule is the detached outboard-limited discharge). 


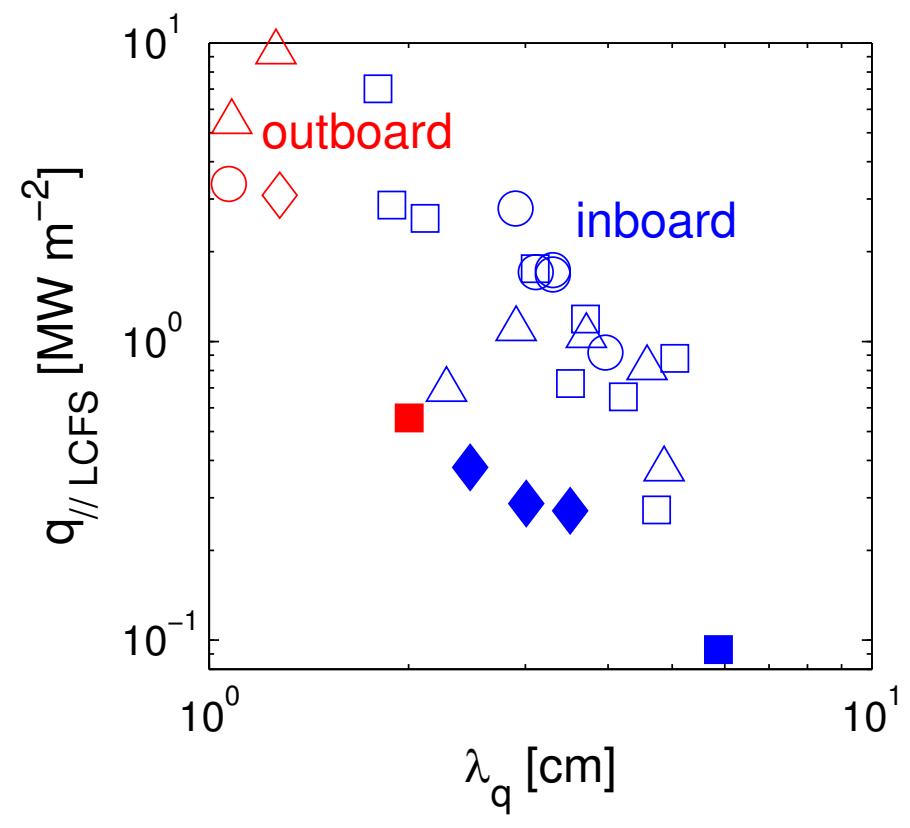

Figure 6. Heat flux density at the LCFS plotted against the SOL heat flux density e-folding length. Inboard / outboard indicates the plasma contact point. Symbols correspond to poloidal cross sections from figure 1. Full symbols: detached discharges.

$q_{/ / L C F S} \propto \lambda_{q}^{-1}$ has a physical background in the conservation of the power in the SOL,

$$
P_{S O L} \approx 4 \pi R \int q_{p} d r \approx 2 \pi R q_{p L C F S} \lambda_{q} \approx R q_{/ / L C F S} \lambda_{q}\left(B_{p} / B_{t o t}\right) \approx q_{/ / L C F S} \lambda_{q} a / q_{a}
$$

where $q_{p}$ is the poloidal component of the parallel heat flux density, $B_{p}$ is the poloidal component of the total magnetic field $B_{t o t}$ and $q_{a}$ is the safety factor at $a$. From Eq. (2) it follows that

$$
q_{/ / L C F S} \approx P_{S O L} q_{a} /\left(a \lambda_{q}\right)
$$

It should be pointed out that the applicability of Eqs. (2) and (3), or even their use as an indication of proportionality, depends on the degree to which the discrete limiters approximate to a toroidally continuous limiter. A rough estimate of the degree to which the limiters in Tore Supra achieve that approximation can be assessed by means of a simple geometrical criterion proposed originally by Stangeby et al. [63] for a set of discrete limiters redistributed equidistantly in the toroidal direction. The discrete outboard limiters will act, in effect, as a toroidally continuous limiter (meaning that all field lines in the SOL strike some outboard (inboard) limiter on their first pass through the outboard (inboard) midplane) if

$$
\frac{\Delta L_{t}}{h_{w}}<\left(\frac{B_{t}}{B_{p}}\right)_{c},
$$

where $\Delta L_{t}$ is the toroidal distance between the limiters, $h_{w}$ is the plasma-wetted height of the limiters, and $\left(B_{t} / B_{p}\right)_{c}$ is the toroidal-to-poloidal magnetic field ratio evaluated at the plasma contact point. For the outboard-limited discharges, (4) was evaluated for $\Delta L_{t}$ given by the 
toroidal distance of the antenna protection limiter and the ICRH limiter located at the toroidal angle of $280^{\circ}$ (i.e. the largest toroidal separation between the outboard limiters, section 2). $h_{w}$ was estimated from $\lambda_{n}$ mapped to the midplane and projected on the outboard limiters. In table 3, the criterion (4) is evaluated individually for each outboard discharge. As seen from the table, the discrete outboard limiters can be approximated by a toroidally continuous limiter for all outboard discharges except \#42400. For the inboard-limited discharges $\left(\Delta L_{t} / h_{w}\right) /\left(B_{t} / B_{p}\right)_{c}<0.05$, so that the inboard bumper limiters also act effectively as a continuous toroidal limiter.

Table 3. A list of the outboard-limited discharges, showing the discharge number, plot symbol and color code used in figures 4-7, volume averaged density, largest toroidal separation of the outboard limiters, plasma-wetted height of the outboard limiters and the toroidal-topoloidal magnetic field ratio at the plasma contact point on the outboard limiters. Last column indicate the degree to which the outboard limiters approximate to a toroidally continuous limiter (see text).

\begin{tabular}{lllllll}
\hline $\begin{array}{l}\text { discharge } \\
\text { number }\end{array}$ & symbol & $\left\langle n_{e}\right\rangle\left[10^{19} \mathrm{~m}^{-3}\right]$ & $\Delta L_{t}[\mathrm{~m}]$ & $h_{w}[\mathrm{~m}]$ & $\left(B_{t} / B_{p}\right)_{c}$ & $\frac{\Delta L_{t}}{h_{w}} /\left(\frac{B_{t}}{B_{p}}\right)_{c}$ \\
\hline 42400 & $\triangle$ & 5.0 & 6.8 & 0.4 & 16 & 1.1 \\
42401 & 0 & 2.2 & 6.8 & 0.5 & 21 & 0.6 \\
42401 & $\triangle$ & 2.4 & 6.8 & 0.4 & 29 & 0.6 \\
42460 & $\diamond$ & 2.0 & 7.9 & 0.9 & 20 & 0.4 \\
42968 & $\square$ & 1.4 & 7.9 & 0.9 & 35 & 0.2 \\
\hline
\end{tabular}

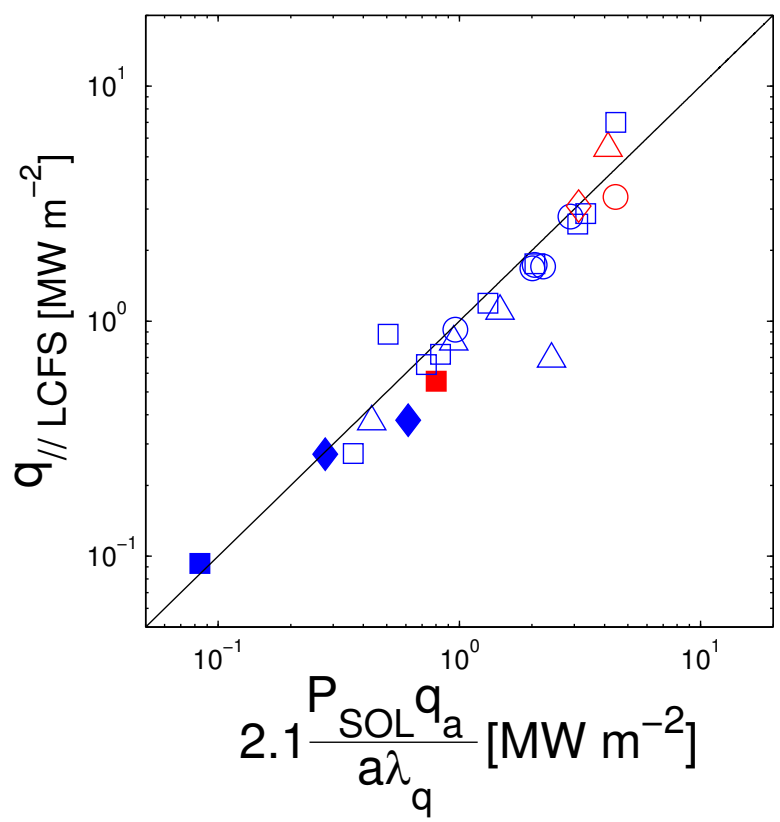

Figure 7. Parallel heat flux density at the LCFS plotted against the physics-based scaling. Constant 2.1 is obtained from the linear least-squares fit for $P_{S O L}$ in $M W$, a in meters and $\lambda_{q}$ in centimeters. Outboard-limited discharge \#42400 is not included in the scaling (see text). 
Figure 7 shows that the physics-based scaling (3) reproduces well $q_{/ / L C F S}$ for both contact points and over a large range of parameters (for the reasons mentioned above the outboard-limited discharge \#42400 is not included in the scaling). $P_{S O L}$ is evaluated as $P_{\text {ohm }}\left(1-f_{\text {rad }}\right)$ with $P_{\text {ohm }}$ being the ohmic input power. In principle, the scaling could be used to predict $q_{/ / L C F S}$ in the ITER start-up plasma with $P_{S O L}, a, q_{a}$ and $\lambda_{q}$ provided by transport analysis [64-66]. The predictive capability of the scaling (3) to ITER is, however, very limited as the database (of the outboard-limited discharges in particular) is statistically insignificant. Another important point to consider is that our measurements were performed in circular plasmas so that the scaling might not be valid for the elongated ITER start-up plasma. In addition, for the outboard-limited discharges a large error on $q_{/ / L C F S}$ should be anticipated because of the uncertainty of the LCFS position as well as the extrapolation of the measured $q_{/ /}$towards the LCFS over the distance comparable to $\lambda_{q}$.

\section{Summary and conclusions}

New measurements were carried out with a RFA in the SOL of Tore Supra to study the poloidal asymmetry of the radial particle and energy transport. The transport asymmetry has been studied by moving the plasma contact point and examining the effect on the SOL profiles of ion and electron temperatures, electron density, and the parallel heat flux density for a broad range of the main plasma parameters. Substantially longer e-folding lengths measured for the outboard- compared to inboard-limited discharges provide again clear evidence that the particle and energy transport across the SOL is enhanced on the outboard side. This was demonstrated for the first time also for the ion energy transport. The results reported here are consistent with similar observations of the poloidally asymmetric particle [8-12, 18-20]) and electron energy [16-20] transport reported earlier.

These results are particularly important for the optimization of the start-up phase and designing the first wall for ITER. A simple physics-based scaling for the parallel heat flux density at the LCFS $q_{/ / L C F S} \propto \lambda_{q}^{-1}$ was found to agree reasonably well with measurements. It suggests that the power handling in ITER start-up phase would be significantly easier if the inboard wall were used for start-up rather than outboard limiters. Systematic measurements of the type presented here, as well as in elongated plasmas, are needed in order to decouple the influence of the individual macroscopic plasma parameters on the e-folding lengths.

\section{Acknowledgement}

The authors acknowledge helpful discussions with Drs. Richard Pitts and François SaintLaurent and the excellent technical assistance of Jean-Yves Pascal. This work, supported by the European Communities under the contract of Association between EURATOM and CEA, was carried out within the framework of the European Fusion Development Agreement. The views and opinions expressed herein do not necessarily reflect those of the European Commission.

\section{References}

[1] Zweben S J 1985 Phys Fluids 28 (3) 974

[2] Krasheninnikov S I 2001 Phys. Lett. A 283368

[3] Garcia O E, Horacek J, Pitts R A, Nielsen A H, Fundamenski W, Graves J P, Naulin V and Juul Rasmussen J 2006 Plasma Phys. Control. Fusion 48 L1-L10 
[4] Garcia O E, Pitts R A, Horacek J, Madsen J, Naulin V, Nielsen A H and Juul Rasmussen J 2007 Plasma Phys. Control. Fusion 49 B47-B57

[5] Garcia O E, Horacek J, Pitts R A, Nielsen A H, Fundamenski W, Naulin V and Juul Rasmussen J 2007 Nucl. Fusion 47 667-676

[6] Naulin V 2007 J. Nucl. Mater. 363-365 24

[7] Asakura N 2007 J. Nucl. Mater. 363-365 41

[8] LaBombard B and Lipschultz B 1987 Nucl. Fusion 2781

[9] Vershkov V A, Grashin S A and Chankin A 1987 J. Nucl. Mater. 145-147 611

[10] Pitts R A, Vayakis G, Matthews G F and Vershkov V A 1990 J. Nucl. Mater. 176-177 893-898

[11] Pericoli Ridolfini V, Zagorski R, Crisanti F, Granucci G, Mazzitelli G, Pieroni L and Romanelli F 1995 J. Nucl. Mater. 220-222 218-222

[12] Gunn J P et al 2007 J. Nucl. Mater. 363-365 484

[13] Tamain P, Ghendrih Ph, Tsitrone E, Sarazin Y, Garbet X, Grandgirard V, Gunn J P, Serre E, Ciraolo G, Chiavassa G 2009 J. Nucl. Mater. 390-391 347

[14] Asakura N, Sakurai S,Itami K, Naito O, Takenaga H, Higashijima S, Koide Y, Sakamoto Y, Kubo H and Porter G D 2003 J. Nucl. Mater. 313-316 820

[15] Asakura N, Takenaga H, Sakurai S, Porter G D, Rognlien T D, Rensink M E, Shimizu K, Higashijima S and Kubo H 2004 Nucl. Fusion 44503

[16] Seigneur A et al 1993 Proc. 20 ${ }^{\text {th }}$ Eur. Conf. Lisbon, Vol. 17C, Part II, European Physical Society, Geneva p 603

[17] Harbour P J and Loarte A 1995 Nucl. Fusion 35759

[18] La Bombard B et al. 2004 Nucl. Fusion 441047.

[19] Keilhacker M and Daybelge U 1981 Nucl Fusion 211497

[20] Smick N 2009 PhD thesis, Massachusetts Institute of Technology

[21] Saoutic B, Chatelier M and De Michelis C 2009 Fusion Sci. Technol. 561079

[22] Staudenmaier G, Staib P, Poschenrieder W 1980 J. Nucl. Mater. 121-126 121

[23] Erents S K and Stangeby P C 1982 J. Nucl. Mater. 111-112 165

[24] Wampler W R and Manos D M 1982 J. Vac. Sci. Technol. A 1 (2) 827

[25] Stangeby P C, McCracken G M, Erents S K, Vince J E and Wilden R 1983 J. Vac. Sci. Technol. A 11302

[26] Matthews G F 1984 J. Phys. D: Appl. Phys. 172243

[27] Wan A S, Yang T F, Lipschultz B and LaBombard B 1986 Rev. Sci. Instrum. 571542

[28] Wan A S et al. 1987 J. Nucl. Mater. 145-147 191

[29] Kimura H et al. 1987 Nucl. Fusion 181195

[30] Höthker K, Belitz H-J, Bieger W and Amemiya H 1990 Rev. Sci. Instrum. 61114

[31] Pitts R A 1990 PhD thesis, University of London

[32] Matthews G F, Pitts R A, McCracken G M and Stangeby P C 1991 Nucl. Fusion 31 1495

[33] Bogen P, Hey J D, Hintz E, Lie Y T, Rusbuldt D and Samm U 1995 J. Nucl. Mater. 220-222 472

[34] Guo H Y, Matthews G F, Davies S J, Erents S K, Horton L D, Mond R D and Stangeby P C 1996 Contrib. Plasma Phys. 3681

[35] Uehara K, Tawakami T, Amemiya H, Höthker K, Cosler A and Bieger W 1998 Nucl. Fusion 381665

[36] Raychaudhuri S 1999 Contrib. Plasma Phys. 39359

[37] Huber A, Pospieszczyk A, Unterberg B, Brix M, Mertens Ph, Philipps V and Schweer B 2000 Plasma Phys. Control. Fusion 42569

[38] Nachtrieb R 2000 PhD thesis, Massachusetts Institute of Technology

[39] Kreter A $2001 P h D$ thesis, Düsseldorf University 
[40] Ratynskaia S V, Demidov V I and Rypdal K 2002 Rev. Sci. Instrum. 734232

[41] Pitts R A, Chavan R, Davies S J, Erents S K, Kaveney G, Matthews G F, Neill G, Vince J E and Duran I 2003 Rev. Sci. Instrum. 744644

[42] Nedzelskiy I S, Silva C, Figueiredo H, Fernandes H and Varandas C A F 2006 Rev. Sci. Instrum. 77 10E729-1

[43] Kočan M, Pánek R, Stöckel J, Hron M, Gunn J P and Dejarnac R 2007 J. Nucl. Mater. 363-365 1436

[44] Adamek J, Kočan M, Panek R, Gunn J P, Martines E, Stockel J, Ionita C, Popa G, Costin C, Brotankova J, Schrittwieser R and Van Oost G 2008 Contrib. Plasma Phys. 481

[45] Kočan M, Gunn J P, Pascal J-Y, Bonhomme G, Fenzi C, Gauthier E and Segui J-L 2008 Plasma Phys. Control. Fusion 50125009

[46] Kočan M, Gunn J P, Komm M, Pascal J-Y, Gauthier E and Bonhomme G 2008 Rev. Sci Instrum. 79073502

[47] Kočan M et al. 2009 J. Nucl. Mater. 390-391 1074

[48] Kočan M 2009 PhD thesis, Nancy-Université

[49] Stangeby P C and Mitteau R 2009 J. Nucl. Mater. 390-391 963

[50] Gribov Y Control System Design and Assessment (CSD), Appendix B: Magnetic configuration and poloidal field scenarios, ITER document N 19 RI 14 R0.1, August 2004.

[51] Litaudon X and Tore Supra Team 2009 Fusion Sci. Technol. 561445

[52] Pégourié B and Tore Supra Team 2009 Fusion Sci. Technol. 561334

[53] Valsaque F, Manfredi G, Gunn J P and Gauthier E 2002 Phys. Plasmas 91806

[54] Hutchinson I H 1987 Phys. Fluids 303777

[55] Dejarnac R, Gunn J P, Stockel J, Adamek J, Brotankova J and Ionita C 2007 Plasma Phys. Control. Fusion 491791

[56] Stangeby P C 2000 The Plasma Boundary of Magnetic Fusion Devices (Institute of Physics Publishing 2000) p 649

[57] Hobbs G D and J A Wesson 1967 Plasma Phys. 985

[58] Wesson J 1997 Tokamaks (Claredon Press - Oxford) p 424

[59] Uehara K et al. 1979 Plasma Phys. 2189

[60] Stangeby P C 1985 J. Phys. D 181547

[61] Moreau Ph, Bremond S, Douai D, Geraud A, Hertout P, Lennholm M, Mazon D, Saint-Laurent F and Tore Supra Team 2009 Fusion Sci. Technol. 561284

[62] Stangeby P C 2000 The Plasma Boundary of Magnetic Fusion Devices (Institute of Physics Publishing 2000) p 160

[63] Stangeby P C, Tagle J A, Erents S K and Lowry C 1988 Plasma Phys. Control. Fusion 301787

[64] Zolotukhin O, Federici G, Tanga A, Loarte A, Horton L, Portone A, Gribov Y, Polevoi A, Shimada M 2006 Proc. $33^{\text {rd }}$ EPS Conference on Plasma Phys. Rome, 1923 June 2006 ECA Vol. 30I, P-1.120

[65] Federici G et al 2007 J. Nucl. Mater. 363-365 346

[66] Kobayashi M et al 2007 Nucl. Fusion 4761 